\title{
Uso del exelearning, aplicación de contenidos digitales y su relación con el proceso de aprendizaje en la Universidad Nacional José Faustino Sánchez Carrión 2017
}

\section{Use of exelearning, application of digital content and its relation to the learning process at the José Faustino Sánchez Carrión National University 2017}

\author{
Uso do exelearning, aplicação de conteúdo digital e sua relação com o processo de \\ aprendizagem na Universidade Nacional José Faustino Sánchez Carrión 2017
}

\author{
${ }^{11}$ Arias Pittman, José Augusto
}

\begin{abstract}
Resumen
La presente investigación, tiene como objetivo analizar si existe relación entre el uso del exelearning con contenidos digitales y su relación con el proceso de aprendizaje. Se trabajó con tres variables Uso de exelearning $(\mathrm{X})$, contenidos digitales $(\mathrm{Y})$ y proceso de aprendizaje $(\mathrm{Z})$. El diseño fue pre experimental de corte longitudinal, con un nivel descriptivo correlacional. Se validó el instrumento a través de cinco juicios de expertos, dándonos una validez del $85 \%$; se probó la confiabilidad de los datos a través del alpha de cronbach's, el cual fue del $94 \%$ de confiablidad. Las cinco hipótesis nulas fueron probadas con la prueba chi cuadrado; las que fueron rechazadas porque se obtuvieron valores estadístico de la prueba entre 0,00 y 0,001; valores menores que el 0,05 de significancia. La prueba pre experimental se hizo a través de la Hipótesis para Muestra Relacionadas, el cual nos determinó que el promedio con aplicación de contenidos digitales fue de 14,69 contra 11,12 sin uso de contenidos digitales. La discusión se hizo con tres tesis doctorales de Rivera (2015), Morales (2013) y Del Águila (2015), las cuales reforzaron nuestra investigación de que si existe relación cuando se hace uso de contenidos digitales con el proceso de aprendizaje. Se concluye que efectivamente la aplicación de contenidos digitales se relaciona con el proceso de aprendizaje cuando se utiliza la herramienta de exelearning.
\end{abstract}

Palabras claves: Contenido digital, exelearning, aprendizaje, plataforma virtual, evaluación en línea

\begin{abstract}
The objective of this research is to analyze if there is a relationship between the use of exelearning with digital content and its relation to the learning process. We worked with three variables Using exelearning $(\mathrm{X})$, digital content $(\mathrm{Y})$ and learning process $(\mathrm{Z})$. The design was pre experimental of longitudinal cut, with a correlational descriptive level. The instrument was validated through five expert judgments, giving us a validity of $85 \%$; the reliability of the data was tested through the cronbach's alpha, which was $94 \%$ confidence. The five null hypotheses were tested with the chi-squared test; those that were rejected because statistical values of the test were obtained between 0.00 and 0.001 ; values less than 0.05 of significance. The pre-experimental test was done through the Related Sample Hypothesis, which determined that the average with application of digital content was 14.69 vs. 11.12 without the use of digital content. The discussion was made with three doctoral theses from Rivera (2015), Morales (2013) and Del Águila (2015), which reinforced our research that if there is a relationship with use of digital content with the learning process. It is concluded that the application of digital content is effectively related to the learning process when the exelearning tool is used.
\end{abstract}

Keywords: Digital content, exelearning, learning, virtual platform, online evaluation

\section{Resumo}

Na presente investigação, seu objetivo é analisar se existe uma relação entre o uso da aprendizagem com conteúdo digital e sua relação com o processo de aprendizagem. Trabalhe com três variáveis Uso do exelearning (X), conteúdo digital $(\mathrm{Y})$ e processo de aprendizado $(\mathrm{Z})$. O delineamento foi em seção longitudinal pré-experimental, com nível descritivo correlativo. Se o instrumento for validado por cinco

\footnotetext{
${ }^{11}$ Universidad Nacional José Faustino Sánchez Carrión. Huacho. Perú. Facultad de Ingeniería Industrial, Sistemas e Informática;.. https://orcid.org/0000-0001-9281-0796
} 
juízes especialistas, com uma validade de $85 \%$; Se a confiabilidade dos dados foi testada através do alfa cronbach, isso representa $94 \%$ da confiabilidade. As cinco hipóteses nulas foram testadas com o quádruplo chi chi; aqueles que foram rejeitados porque foram obtidos os valores estatísticos da pressão entre $0,00 \mathrm{e}$ 0,001; valores menores que le 0,05 de significância. O estudo pré-experimental foi realizado através da Hipótese para Assuntos Relacionados, conforme determinado pelo fato de que a promessa da aplicação do conteúdo digital foi de 14,69 versus 11,12 com o uso de conteúdo digital. A discussão é realizada com três teses de doutorado de Rivera (2015), Morales (2013) e Del Aguila (2015), o que reforça nossa investigação de que existe uma relação quando o conteúdo digital é usado com o processo de aprendizagem. Conclui-se que efetivamente a aplicação do conteúdo digital está relacionada ao processo de aprendizagem quando a ferramenta de aprendizagem é utilizada.

\section{Palavras chave}

Conteúdo digital, e-learning, aprendizado, plataforma virtual, avaliação online.

\section{Introducción}

El presente trabajo tiene como propósito analizar cómo el Exelearning con aplicación de contenidos digitales se relaciona con el proceso de aprendizaje en la Universidad Nacional José Faustino Sánchez Carrión 2017. La presente investigación se hace con la finalidad de demostrar, si los contenidos digitales tiene influencia en el proceso de aprendizaje de los estudiantes del VI ciclo del curso de Ingeniería de Métodos y Ergonomía, de la Escuela de Ingeniería Industrial de la Universidad Nacional José Faustino Sánchez Carrión de Huacho; estos contenidos se diseñan con anticipación, y antes de entrar a la clase presencial, se les envía el archivo digital, a través de la plataforma virtual para que tomen conocimiento del contenido y de lo que se tratará en dicha clase, teniendo inclusive una autoevaluación con fines de retroalimentación, comunicándoles a los estudiantes que el tiempo de cierre del archivo es un día antes de la clases programadas en el sílabo.

\section{Métodos}

La investigación se realizó en dos momentos diferentes, pero al mismo grupo, en los dos primeros meses, se les enseñó en forma tradicional, con utilización de pizarra, equipos de multimedia y con exámenes presenciales, a todos los estudiantes de ese ciclo, eso significa el momento antes; luego en los dos meses siguientes se les entregó los archivos digitales para que los estudiantes los puedan estudiar antes de entrar a clase; eso significa el momento después. Por otro lado la investigación corresponde a un diseño pre experimental de la situación actual de cómo los contenidos digitales tiene relación con el aprendizaje de los estudiantes del curso de Ingeniería de Métodos y Ergonomía, utilizando como herramienta el exelearning y teniendo como soporte la plataforma virtual de enseñanza de la Facultad de Ingeniería Industrial. La población fue de 35 estudiantes que cursan esta asignatura, y la muestra fue la misma que la población, es decir censal, la validez de contenido del instrumento se hizo a través de juicios de expertos de cinco catedráticos de la Escuela de Pos Grado de la Universidad Nacional de Educación, dando un coeficiente de contenido de $85 \%$, considerado como Muy Bueno, así mismo la validez de criterio se hizo a través de Alpha de Cronbachs, el cual nos dio una confiablidad del $94 \%$, por lo que consideramos que el instrumento es muy confiable. El estudio se ha realizado en el VI ciclo de la Escuela de Ingeniería Industrial de la Universidad Nacional José Faustino Sánchez Carrión, y para la obtención de las apreciaciones de los estudiantes, se utilizó una encuesta con 25 preguntas.

\section{Resultados}

Un resultado importante encontrado fue que el valor estadístico de la prueba $\boldsymbol{p}$, es de 0,00 para ambos pares, y este valor es menor al nivel de significancia $\boldsymbol{\alpha}$ del $5 \%(0,05)$, por lo tanto rechazamos la Ho y aceptamos la H1, eso significa que hay evidencias para aceptar la hipótesis alternativa, que el promedio de las variables uso de exelearning, aplicación de contenidos digitales es diferente a la variable promedio del proceso de aprendizaje. En otras palabras afirmamos que la enseñanza con contenidos digitales utilizando exelearning si tiene incidencia en el aprendizaje de los estudiantes. Para demostrar lo que afirmamos, determinamos los promedios generales de cada módulo, y se aprecia que ha sido ascendente desde el PM1 (Promedio del Módulo I) de 11,220 hasta el PM4 (Promedio del Módulo IV) 14,074; valores, que reafirman, que los contenidos digitales han incidido en el mejor aprendizaje de los estudiantes. 


\begin{tabular}{|c|c|c|c|c|c|c|c|c|c|}
\hline \multicolumn{10}{|c|}{ Paired Samples Test } \\
\hline & & \multicolumn{5}{|c|}{ Paired Differences } & \multirow[b]{3}{*}{$\mathrm{t}$} & \multirow[b]{3}{*}{ df } & \multirow[b]{3}{*}{ Sig. (2-tailed) } \\
\hline & & \multirow[b]{2}{*}{ Mean } & \multirow[b]{2}{*}{ Std. Deviation } & \multirow{2}{*}{$\begin{array}{l}\text { Std. Error } \\
\text { Mean }\end{array}$} & \multicolumn{2}{|c|}{$\begin{array}{l}\text { 95\% Confidence Interval of the } \\
\text { Difference }\end{array}$} & & & \\
\hline & & & & & Lower & Upper & & & \\
\hline Pair 1 & $\begin{array}{l}\text { Promedio Modulo I- } \\
\text { Promedio Modulo III }\end{array}$ & $-2,2429$ & 1,3448 &, 2273 & $-2,7048$ & $-1,7809$ & $-9,867$ & 34 &, 000 \\
\hline Pair 2 & $\begin{array}{l}\text { Promedio Modulo II- } \\
\text { Promedio Modulo IV }\end{array}$ & $-2,4400$ & 8630 & 1459 & $-2,7365$ & $-2,1435$ & $-16,726$ & 34 &, 000 \\
\hline
\end{tabular}

Figura 1 Prueba t para muestras relacionadas antes (Módulo I y II) y después (Modulo II y IV).

\section{PROMEDIO DE LOS CUATRO MÓDULOS}

16.000

14.000

12.000

10.000

8.000

6.000

4.000

2.000

0.000

- Series1

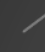

\section{PROMEDIO I}

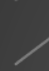

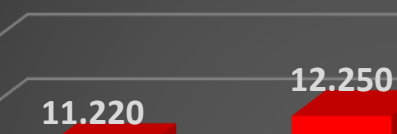

\subsection{0}

14.690

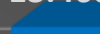


Seminario de especialidad de la Facultad de Ciencias - Universidad Nacional de Educación Enrique Guzmán y Valle-2012", llegó a la conclusión de que; "Se puede inferir con un nivel de significación de 0.05, que el nivel de aprendizaje de los estudiantes del X ciclo de Informática que cursan Seminario de especialidad antes de la aplicación del Moodle como sistema de gestión de aprendizaje de la Facultad de Ciencias de la Universidad Nacional de Educación Enrique Guzmán y Valle - 2012 es bajo. Asimismo, infieren que, con un nivel de significación de 0.05, el nivel de aprendizaje de los estudiantes del X ciclo de Informática que cursan Seminario de especialidad después de la aplicación del Moodle como sistema de gestión de aprendizaje de la Facultad de Ciencias de la Universidad Nacional de Educación Enrique Guzmán y Valle - 2012 es alto; lo cual, reafirma nuestra investigación de que los estudiantes del VI ciclo del curso de Ingeniería de Métodos y Ergonomía, tienen un promedio ponderado en el antes, menor, mientras que el proceso después es mayor.

\section{Conclusiones}

La presente investigación llegó a la conclusión que el uso de exelearning, aplicación de contenidos digitales y su relación con el proceso de aprendizaje en la Universidad Nacional José Faustino Sánchez Carrión 2017, resultaron diferentes, ya que con la aplicación de la prueba pre experimental demuestra que los rendimientos académicos de la prueba después fueron mayores (M3 13,46 y M4 14, 69) a los rendimientos de la prueba antes (M1 11,22 y M2 12,25). En cuanto al uso de exelearning se relaciona con el proceso de aprendizaje en la Universidad Nacional José Faustino Sánchez Carrión 2017, a un nivel de significancia del $5 \%$, la prueba de independencia nos demostró que si hay relación entre el uso del exelearning y el proceso de aprendizaje, ya que es una herramienta que permite diseñar contenidos académicos digitales que son muy apreciados por los estudiantes. Con respecto a la aplicación de contenidos digitales se relación con el proceso de aprendizaje en la Universidad Nacional José Faustino Sánchez Carrión 2017, a un nivel de significancia del $5 \%$, la prueba de independencia nos demostró que si hay relación entre la aplicación de los contenidos digitales y el proceso de aprendizaje, porque estos contenidos académicos digitales presentan textos, videos, imágenes, sonido de voz, que hacen más atractivo el aprendizaje de los estudiantes.

\section{Referencias}

Álvarez S. (2010). Uso de contenidos educativos digitales a través de sistemas de gestión del aprendizaje (lms) y su repercusión en el acto didáctico comunicativo. Universidad Complutense Madrid. Memoria para optar al grado de doctor. Recuperado http://eprints.ucm.es/11631/1/T32372.pdf.

Bernal, L. (2010). Fundamento tecno pedagógico en e-learning. Disponible en https://earchivo.uc3m.es/handle/10016/9154\#preview. Consultado el 6 de diciembre del 2017.

Bueno, G. (2010). Modelo de repositorio institucional de contenido educativo (RICE): la gestión de materiales digitales de docencia y aprendizaje en la biblioteca universitaria. Universidad Carlos III de Madrid. Disponible en www.luisbernal.es. Consultado el 22 de junio de 2010.

Chiappe A, (2016). Tendencia sobre contenidos educativos digitales en América Latina. España. . p. 52, 56-57.

Cubillo J. (2014). Arle: una herramienta de autor para entornos de aprendizaje de realidad aumentada. Tesis Doctoral. UNED. España.

Del Águila V. (2015). Uso de la plataforma virtual y su influencia en el rendimiento académico en los módulos de Gestión Agrícola de los estudiantes del Instituto Superior Tecnológico Privado "Valle Grande" de Cañete - 2014 Lima. Perú. (p 12, 81).

Díez O. (2007). "El reto de la nueva educación a distancia: enseñar a los alumnos a aprender en red" En: Jerónimo, JA. (2007). Aprendizaje en RED, en busca de la comunidad virtual. México: FES Zaragoza UNAM. 
Dorado C. (2010). El diseño de contenidos multimedia para entornos virtuales de aprendizaje. Disponible en http:// mem.uab.cat/cdorado/Articulos/DISENO_\%20CONTENIDOS_\%20MULTIMEDIA.pdf Consultado el día 22 de julio de 2010.

Finger, G. (2013). Teaching teacher for the future project building TPACK confidence and capabilities for elearning. Australia. Fonseca, M. (2013). Revista de educación mediática y TIC. EDMETIC.

García A. (2012). Contenidos digitales un complemento a los libros de textos. España. Recuperado de http://www.consumer.es/web/es/educacion/escolar/2012/11/09/214069.php

Gonzales L. (2014). Influencia del uso del Aula Virtual en el Nivel de Autoaprendizaje e Integración Social en Estudiantes del Colegio de la FAP Víctor Maldonado Begazo Nº1104 de Magdalena Del Mar. Lima - Perú (P 68, 108)

Hernández R., Fernández C. y Baptista P. (2014). Metodología de la investigación. Sexta Edición. Editorial Mc Graw Hill Education. México.

Herrera L., Maldonado G, Mendoza N, Pérez E. (2007). Metodología para el diseño de material didáctico en plataforma de e-learning. I Congreso Internacional Escuela y TIC. Alicante.

Infante G, Pareja V, Silva P. (2015). Uso de contenidos educativos digitales a través de sistema de gestión del aprendizaje (LMS) y su repercusión en el acto didáctico comunicativo. Universidad Complutense de Madrid.

Jáuregui G. (2016). Aplicación del aula virtual y su influencia en el aprendizaje del curso de informática de los estudiantes del segundo ciclo de la Universidad Alas Peruanas-UAD Chosica, Lima - Perú 2016 (p4, 100).

.Mayorga M, Alfonso D, Escamillas R, (2012) Lineamientos comunicativos para el aprendizaje en ambientes virtuales. Centro de Apoyo para el Aprendizaje Virtual. Universidad Antonio Nariño. Marzo, 2012. Bogotá, Colombia.

Morales G. (2013) el Moodle como sistemas de gestión de aprendizaje (lms) y su influencia en el aprendizaje de los estudiantes del x ciclo de informática de seminario de especialidad de la Facultad de Ciencias - Universidad Nacional de Educación Enrique Guzmán y Valle2012. Lima. (p8, 117)

Palomino J. (2011). Corrientes pedagógicas contemporáneas UNE. Recuperado de un ppt. Lima.

Pantoja H. (2015). Aplicación del software libre SAGE y su influencia en el rendimiento académico en cálculo vectorial, en los estudiantes del IV ciclo de Ingeniería Mecánica de la Universidad Nacional de Ingeniería. Lima Perú. (p 19, 110)

Perrusquia, E (2009). The influence and shaping of digital technologies on the learning-and learning trajectories-of mathematical concepts. (pp. 179-226). Springer US.

Prieto M, Mendez V, Pech S y Brito J. (2011). VIII Multidisciplinary symposium on design and evaluation of digital content for education. Pedagogical applications of Information Technologies and Communication in Primary Education: Using the interactive whiteboard in educational contexts. España

Rivera E. (2015). Efectos de la tecnología de la información y de las comunicaciones en el aprendizaje de la ingeniería de costos en la Facultad de Ingeniería y Arquitectura de la Universidad San Martín de Porres. Lima- Perú. (p81, 129)

Rodríguez J, Escofet A. (2006). Aproximación centrada en el estudiante como productor de contenidos digitales híbridos. Revista Universidad y Sociedad del Conocimiento; 2:2028. www.uoc.edu/rusc

Sangrá, A. (2005) Los materiales de aprendizaje en contextos educativos virtuales: Pautas para el diseño tecno pedagógico. Barcelona: UOC.

Santiso M; González, Begoña; (2005). Diseño multimedia en e-learning para el ámbito universitario. Revista No Solo Usabilidad, $\mathrm{n}^{\circ}$ 4, 2005. Disponible 
en www.nosolousabilidad.com/articulos/multimedia_elearning.htm. Consultado el 27 de julio de 2010.

Seoane A, García F, Bosom A., Fernández E., Hernández M. (2006). Online tutoring asquality guarantee on elearning based lifelong learning. Definition, modalities, methodology, competences and skills. Virtual Campus. Selected and Extended Papers. CEUR Workshop Proceedings, 186, 41-55.

Sindey C, Bernal V. (2015). Diseño y Creación de Contenidos Educativos Digitales a través de las Herramientas Web 2.0. Recuperado http://www.tise.cl/volumen11/TISE2015/464468.pdf.

Siddip F, (2016). A comprehensive inquiry of the educational readiness for the digital era (Una investigación exhaustiva de la educación preparación para la era digital). University of Oslo

Universidad de Oviedo (2014). Manual de exelearning (Herramienta de autor para creación de contenidos. España. 